\title{
Aeromechanics of membrane and rigid wings in and out of ground-effect at moderate Reynolds Numbers
}

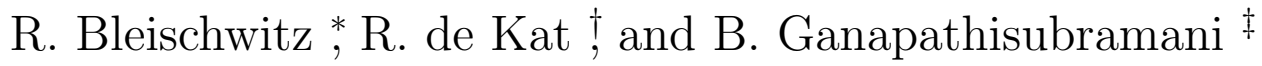 \\ Aerodynamics \& Flight Mechanics Group \\ Faculty of Engineering and the Environment \\ University of Southampton \\ Southampton, SO17 1BJ, UK
}

February 2016

\begin{abstract}
Wind tunnel experiments are conducted using membrane wings and rigid flat-plates in ground-effect at a moderate Reynolds number of $R e=56,000$ with ground clearances from $1 \%$ to $200 \%$ chord length measured from their trailing-edge. A six-axis load-cell captures time-resolved forces and moment while time-resolved stereo digital image correlation (DIC) measurements are performed to capture membrane motions. The lift and drag coefficients of the rigid wing in ground-effect follow well-established trends while the membrane wing appears to exhibit improved coefficients and efficiency (compared to the rigid wing) when in ground-effect. Proper orthogonal decomposition (POD) is applied to study the spatiotemporal structure of membrane vibrations. With increasing angles-ofattack and/or decreasing heights above ground, mode shapes of membrane deformation are dominated by large-scale fluctuations that have a smaller number of local extrema along the chord. Ground-effect induces modifications to the membrane deformation, which appear to be similar to the modifications induced by increasing angles-of-attack in free-flight. At high angles-of-attack in free-flight or at moderate angles in ground-effect, two POD modes of membrane fluctuations are found to be sufficient to capture $90 \%$ of all membrane deformations. Under these conditions, a membrane deformation with maximum camber near the trailing edge of the membrane wing is found to correlate with high lift, low drag and a nose down pitching moment. The extrema in membrane deformations and lift and drag forces occur simultaneously, while there is a time-lag between the deformation and the pitching moment.
\end{abstract}

\footnotetext{
${ }^{*}$ PhD-Student: R.Bleischwitz@soton.ac.uk

${ }^{\dagger}$ Research Fellow: R.De-Kat@soton.ac.uk

¥Professor: G. Bharath@soton. ac.uk
} 


\section{Nomenclature}

\begin{tabular}{llll}
$\alpha$ & global angle-of-attack $\left[^{\circ}\right]$ & $C_{L} / C_{D}$ aerodynamic range efficiency \\
$\beta$ & membrane contact angle $\left[^{\circ}\right]$ & $\left(C_{L} / C_{D}\right)^{\prime}$ fluctuation in $C_{L} / C_{D}$ ratio \\
$\Pi$ & aeroelastic parameter $\left(\frac{E t}{q c}\right)^{1 / 3}$ & $C_{M / 4}$ & pitch coefficient at $1 / 4$ chord \\
$\sigma$ & standard deviation & $h$ & height above ground \\
$\phi$ & phase angle of correlation $\left[^{\circ}\right]$ & $L E / T E$ & leading/trailing edge \\
$a$ & POD coefficient & $P O D$ & proper orthogonal decomposition \\
$c$ & chord length & $P S D$ & power spectral density \\
$C C F$ & cross correlation function & $q$ & dynamic pressure $\frac{1}{2} \rho U_{\infty}^{2}$ \\
$C_{D}$ & drag coefficient & $R e$ & Reynolds number $\frac{U_{\infty} c}{f_{c}^{\nu}}$ \\
$C_{D}^{\prime}$ & drag coefficient fluctuation & $S t$ & Strouhal number $\frac{f U_{\infty}}{U_{\infty}}$ \\
$C_{L}$ & lift coefficient & $z$ & camberwise deflection \\
$C_{L}^{\prime}$ & lift coefficient fluctuation & $z^{\prime}$ & camberwise fluctuation \\
& & & \\
& & & \\
\hline
\end{tabular}

\section{INTRODUCTION}

Increasing interest in Micro-Air-Vehicles (MAVs) has pushed research and adaption of bioinspired membrane wing structures (Swartz et al., 2007), since these thin and flexible membrane wings can improve aerodynamic flight performance at moderate Reynolds numbers $(R e=$ $\left.10^{4}-10^{5}\right)$. Membrane wings combine vehicle agility, enlarged maximum stall angle, extended maximum lift and smooth gust reaction (Ifju et al., 2002; Stanford et al., 2014; Shyy et al., 1999; Lian et al., 2003). The main source of their benefits is suggested to rely on their inherent membrane oscillations which can energize the weak boundary layer to enforce longer flow attachment (Rojratsirikul et al., 2010). In addition, membrane vibrations are found to enhance leading edge vortex shedding, forming large vortices that can significantly contribute to the total lift enhancement at high incidence. The key to understanding the aeromechanics of flexible membrane wings is to understand the coupling effects between flow, membrane and force dynamics (Bleischwitz et al., 2014; Lian et al., 2003).

At low to moderate angles-of-attack, the dynamics of membrane wings come at a price. The aerodynamic efficiency of membrane wings is found to be restricted in comparison to rigid flat plates due to higher drag penalties (Timpe et al., 2013; Galvao et al., 2006; Bleischwitz et al., 2015b). Recent attempts focused on active control of membrane wing performance by the use of electroactive membranes, allowing to gain control over mean camber adjustment or even modulating membrane vibrations (Curet et al., 2014; Hays et al., 2012). However, this challenging concept would add large electro-mechanic complexity in a MAV structure and most likely comes with a payload penalty.

Operating membrane wings close to a surface could be one further option to gain efficiency whilst maintaining the aerodynamic and weight benefits of (passive) membrane wings. A wing is said to be in moderate ground-effect if its height from the ground is either lower than half 
of the wingspan $h / b<0.5$ or below one chord length $h / c<1$ (Yun et al., 2010), where the height-over-ground is measured from the trailing edge of the airfoil and normalised with chord length.

Ground-effect of common thick airfoils relies on the increase in pressure below the wing which leads to a drop in velocity and results ultimately in a downwards movement of the stagnation point (Ahmed and Sharma, 2005). Extreme ground-effect is defined as $h / c<0.1$, where air is forced to stagnate below the wing, causing a large pressure increase, called ram-pressure. As a result, the flow is diverted over the upper wing surface. A wing's effective aspect ratio is increased in ground-effect as the tip vortices are pushed outwards. The interference of the tip vortices with the ground slows down their rotation, accompanied with a reduction in tip vortex size and strength (Han and Cho, 2005). As a result, the downwash angle and induced drag are found to decrease in ground-effect, allowing significant benefits in aerodynamic efficiency of up to $100 \%$ (Rozhdestvensky, 2006).

The vast majority of ground-effect research has focused on high Reynolds number flows $R e>10^{5}$ passing over thick and rigid wings (Qu et al., 2014, 2015) and their application on large Wing-In-Ground (WIG) vehicles (Van Beek and Oskam, 1998; Carter, 1961; Lee et al., 2011), Formula-one aerodynamics (Zhang and Zerihan, 2003; Diasinos et al., 2012), or trains (Moon et al., 2005). A reduction in airfoil thickness is found to benefit (lift producing) wings in groundeffect by reducing drag and lowering the risk of negative down-force caused by the Venturi effect (suction on lower concave surface) (Rad and Kazemi, 2001; Moon et al., 2005). An increase in wing camber is found to improve aerodynamic efficiency within ground proximity, but shows also a higher risk of flow separation on the suction side of the wing (Mosaad et al., 2011), which results in stronger limitations in flight incidences where high aerodynamic efficiency can be achieved.

Studies on Reynolds number influence reveal a reduction in lift coefficient and aerodynamic efficiency with lower Reynolds numbers, which appear even more severe for small ground clearances (Hsiun and Ghent, 1996; Mateescu et al., 2012). Most studies of rigid wings in ground-effect at low to medium Reynolds number date back to the early 20th century and focus on time-averaged aerodynamic performance (Raymond, 1921; Wieselsberger, 1921; Pistolesi, 1937). A recent computational study at $R e=15,000$ concentrated more on the dynamics and found enlarged amplitudes in lift oscillation and tip-vortex-ground interactions within close proximity to the ground (Prasad and Damodaran, 2013).

The physics of flexible membrane wings in ground-effect still remain unknown, however, they will be important for the development of a membrane wing Wing-In-Ground MAV/UAV, aiming to combine improved vehicle range (ground-effect related) and smooth gust reaction (membrane related). Therefore, the current experimental study focuses on understanding the fluid-structure-ground interaction of rectangular perimeter-reinforced membrane wings at a moderate Reynolds number $(R e=56,000)$. The aerodynamic performance of the membrane wings is compared with a rigid flat plate. Instantaneous membrane deformations are measured with Digital-Image-Correlation (DIC) and are compared with simultaneously recorded force and moment dynamics. We consider static and dynamic modifications in lift, drag, and pitch coefficients, their fluctuation intensity and their spectral content. Time-resolved membrane motions are used to extract membrane mean deflection, fluctuation intensity, mode development and their spectral content in the vicinity of the ground. Lift, drag and pitching moment are correlated with membrane motions to find coupling effects between membrane deformation and the coefficients. Finally, Proper-Orthogonal-Decomposition (POD) analysis is applied on 
membrane oscillations to determine the spatiotemporal evolution of the membrane motions.

\section{EXPERIMENTAL SETUP}

\subsection{Wind tunnel}

Wind tunnel experiments are conducted in an open-loop, low-speed blow-down wind tunnel at the University of Southampton. The test section measures $43 \mathrm{~cm}$ width by $48 \mathrm{~cm}$ height with a free-stream turbulence intensity of less than $0.1 \%$. A wind speed of $8.4 \mathrm{~m} / \mathrm{s}$ results in a chord related Reynolds number of $R e=56,000$. The wind speed is determined by measuring dynamic pressure with a pitot-static probe together with barometric pressure and ambient temperature, where the latter two are used to determine the air density $\rho$. The uncertainty in the free-stream velocity is $\pm 0.1 \mathrm{~m} / \mathrm{s}$.

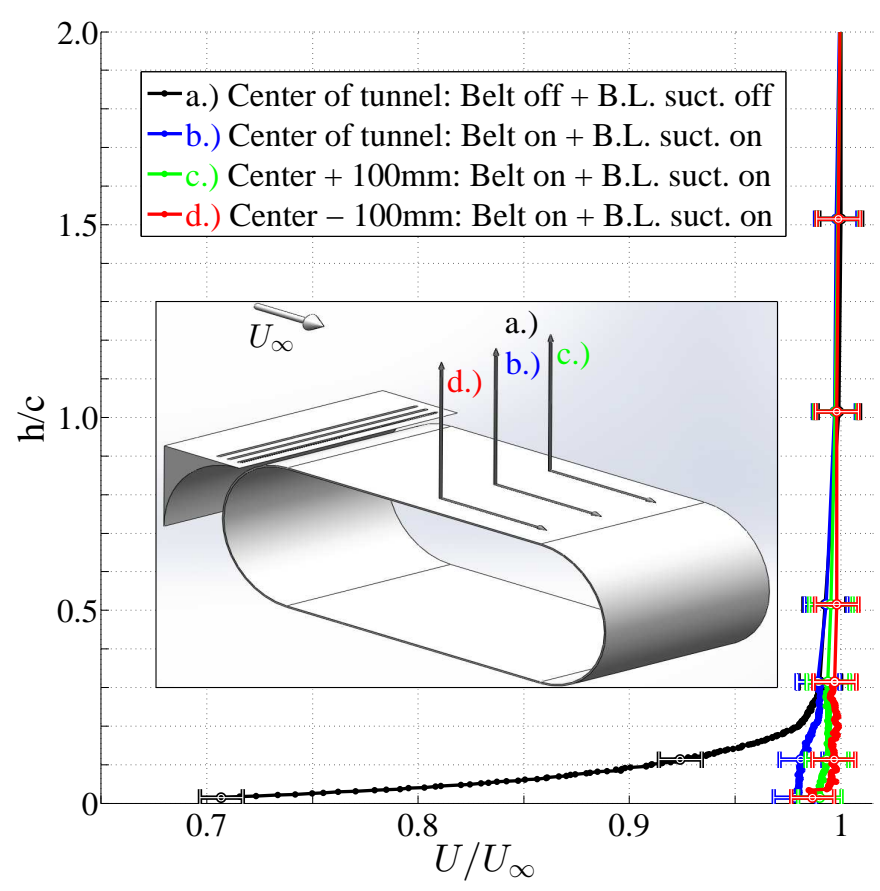

Fig. 1 A rolling road system removes the boundary layer and ensures a uniform boundary layer profile with deviations of less than $2 \%$ from the free-stream velocity.

Without the usage of the rolling road system, the velocity at the wind tunnel wall declines to $70 \%$ of the free-stream-velocity $1.5 \mathrm{~mm}(\mathrm{~h} / \mathrm{c}=0.015)$ above the ground (Figure 1 , black curve). Therefore, a rolling road system is used to simulate correct ground-effect conditions. The belt speed matches the free-stream velocity and boundary layer suction is applied with air slots in front of the $300 \mathrm{~mm}$ wide belt, enabling a nearly uniform, vertical velocity-profile, reaching $98 \%$ of the free-stream-velocity $1.5 \mathrm{~mm}(\mathrm{~h} / \mathrm{c}=0.015)$ above the ground in the midspan position (Figure 1, blue curve). In addition, the boundary layer was measured offset by $100 \mathrm{~mm}$ in wing-span direction on either side of the wind tunnel center (Figure 1, green+red curve). The error-bars indicate that the spanwise velocity variations stay small and within the overall measurement uncertainty of $\pm 0.1 \mathrm{~m} / \mathrm{s}$. A streamlined sting system is mounted from 
the top of the wind tunnel, enabling changes in height-over-ground and angle-of-attack of the wing. Further details of the system can be seen in Bleischwitz et al. (2015a).

Experiments are carried out for relative ground clearances ranging from $0.01 \leq h / c \leq 2$ (relative to the trailing edge), where the height $h / c=2$ represents the center of the wind tunnel test section with free-stream flow conditions. The exact measurement points are $h / c=[0.01$, $0.02,0.05,0.1,0.2,0.3,0.4,0.5,0.75,1,1.5,2]$. In addition, the angle-of-attack is varied between $0^{\circ} \leq \alpha \leq 40^{\circ}$ with a step size of $2.5^{\circ}$.

\section{$2.2 \quad$ Wing design}

The full wing models have a rectangular planform with a chord length of $100 \mathrm{~mm}$ and a wingspan of $200 \mathrm{~mm}$, resulting in an aspect ratio of $\mathrm{AR}=2$ (Figure 2). The wingspan to tunnel width ratio for this span is 0.47 , which is well below a maximum of 0.8 suggested by (Barlow et al., 1999) to avoid significant wall effects.

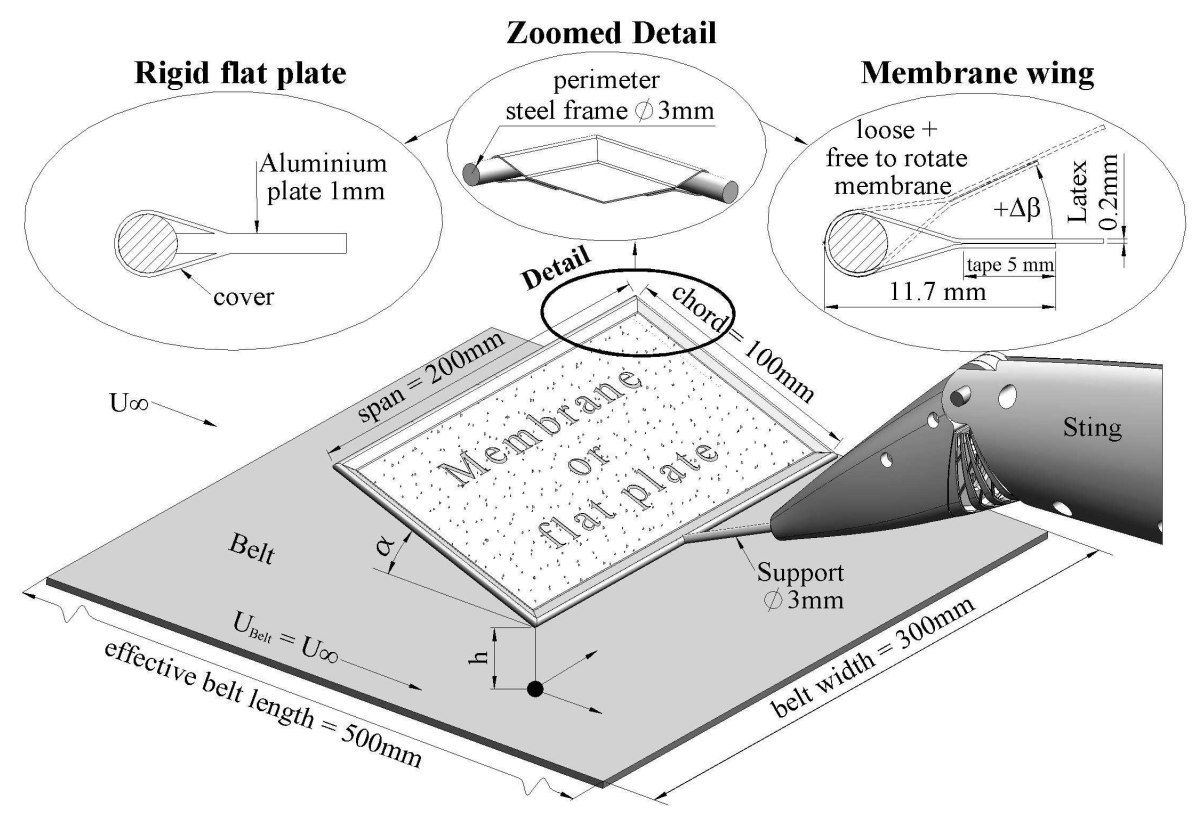

Fig. 2 Sting system with illustrated wing layout for rigid flat plate and membrane wing.

The rigid flat plate consist of a $1 \mathrm{~mm}$ thin aluminium plate which is surrounded by a $3 \mathrm{~mm}$ diameter perimeter steel frame. The transition between steel frame and the aluminium plate is aerodynamic covered to be comparable with the edge design of the membrane wing models (Figure 2, top left).

The perimeter reinforced membrane wing consists of a latex membrane and a steel frame. The latex sheet material has a thickness of $\mathrm{t}=0.2 \mathrm{~mm}$, density of $1 \mathrm{~g} / \mathrm{cm}^{3}$ and a stiffness of $\mathrm{E}$ $=1.5 \mathrm{MPa}$. The aeroelastic parameter $\Pi=(\mathrm{Et} / \mathrm{qc})^{1 / 3}$ (Smith and Shyy, 1996) is found to be $\Pi=4.27$ for $U_{\infty}=8.4 \mathrm{~m} \cdot \mathrm{s}^{-1}$. The membrane was wrapped around the $3 \mathrm{~mm}$ perimeter steel frame and attached to itself with a $5 \mathrm{~mm}$ wide and $0.05 \mathrm{~mm}$ thin double sided tape (Figure 2, top right). 
The $3 \mathrm{~mm}$ supports were selected based on the findings of Arbos-Torrent et al. (2013), who found that larger support diameters $(5 \mathrm{~mm}$ instead of $3 \mathrm{~mm}$ ) can act more like a bluff body and can promote (unwanted) larger separation bubbles, causing a steeper lift incline, accompanied with earlier and sharper stall conditions. In contrast, the smaller $3 \mathrm{~mm}$ support was found to maintain a smoother lifting behaviour beyond stall and encourages the dynamic response of membranes to a larger extent. In addition, sharp instead of round leading edge shapes were found to excite the interference between the rigid wing support structure and the membrane, which coupling was not in focus of the current study.

Assembly of the membrane wing is conducted using a custom made aluminium frame that supports the thin membrane in mid plane of the perimeter steel frame during assembly ensuring a maximum excess length of $\epsilon / c<0.01$ of the membrane and no pre-tension (nominally). The wrapped attachment method of the membrane allows to change contact angles $\beta$ of the membrane on the outer perimeter steel frame (rotation around LE, TE and both wing tips, with slight constrains at the corners, see Figure 2).

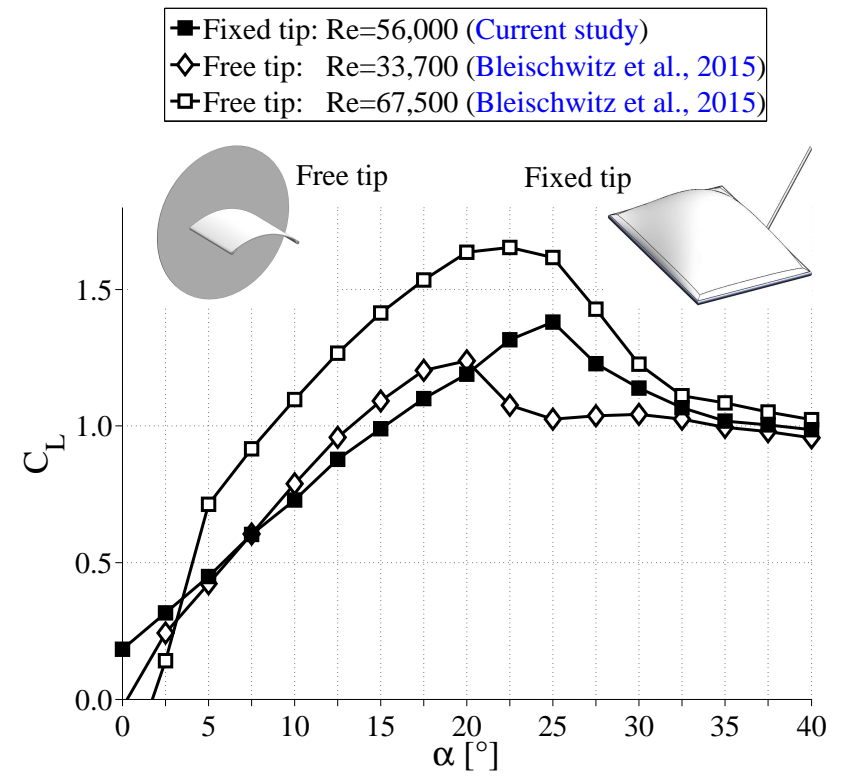

(a) Lift

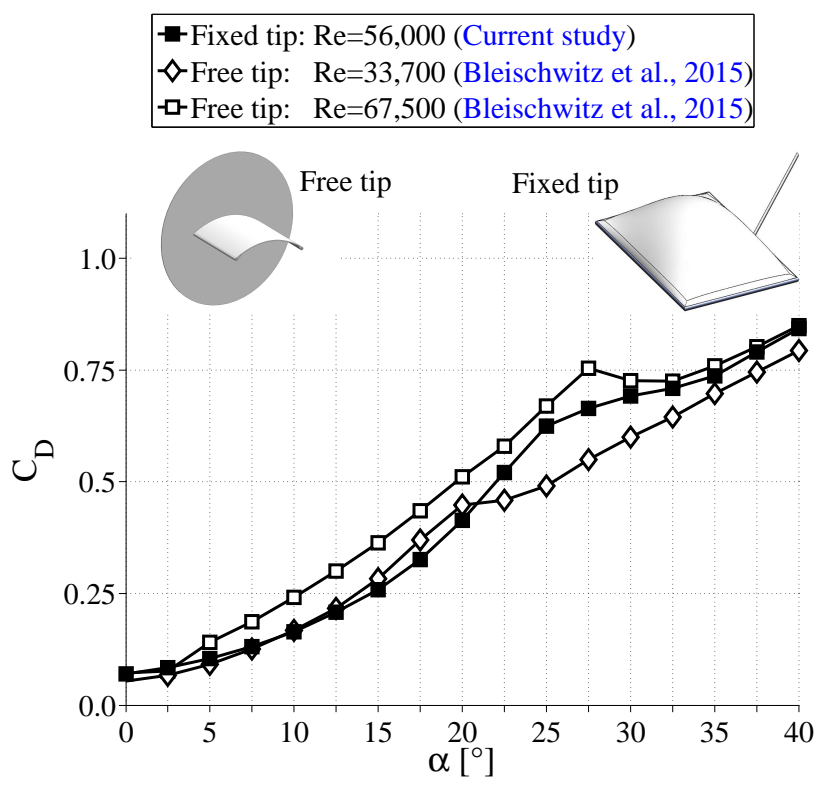

(b) Drag

Fig. 3 Aerodynamic effect of side (tip) support fixation on membrane wings.

A previous study on membrane half wings with unsupported wing tips (membrane free to billow up at wing-tip) showed that the usage of free-to-rotate leading and trailing edges allowed smoother shape adaption, resulting in higher stall angles and gain in maximum lift (Bleischwitz et al., 2014, 2015b). The current study uses a similar free-to-rotate membrane attachment concept, however the membrane is additionally constrained at the wing tips due to the perimeter design. The aerodynamic comparison of a membrane wing with a free and fixed wing tip attachment (Figure 3) shows that the constrained perimeter wing-tip concept of the current study comes with a significant reduction in lift slope. Since the Reynolds number of the current study $R e=56,000$ lies between the Reynolds numbers of the reference cases of $R e=33,700$ and 67,500 , and the lift slope of the current study is shallower than both the reference studies, the difference can be attributed to the difference in free and fixed wing tip. For the perimeter wing-tip concept the membrane is more constrained and cannot freely billow 
up at the wing tips. As a result, the overall mean camber is limited to grow, resulting in a lift penalty. The membrane with a fixed tip also exhibits a lower drag (most likely due to a reduction in induced drag).

\subsection{Force/moment measurement}

All force and moment measurements are made for five different membrane wing samples and the mean loads and moments are computed from the resulting data. The attachment error has very little effect on the membrane dynamics as the force/moment spectra show almost identical dominant frequencies for a given angle-of-attack and height. The overall standard deviation ( $1 \sigma=68 \%$ confidence level) is computed by pooling the data from the five different samples.

Lift and drag forces are measured by a six-axis load cell (ATI-Nano 17) with a maximum load capacity of $25 \mathrm{~N}$. A sampling rate of $10 \mathrm{kHz}$ and a sample length of $20 \mathrm{~s}$ was used. The uncertainty in the load cell is $\pm 0.006 \mathrm{~N}$ in forces and $\pm 0.03[\mathrm{Nmm}]$ in pitching moment (ATI intern calibration, ensured by ISO 9001). However, this accuracy is found to hold only if the measurements were made within a overall time frame of 60 s due to effect of electrical drift (and or temperature related) of the load cell. In order to ensure reliable measurements, a wind-off tare point is obtained as a baseline just prior to the each measurement for all combinations of angle-of-attack and ground height. As a result, an individual membrane wing sample shows an uncertainty in drag of less than $3 \%$ at $\alpha=0^{\circ}$ and $<1 \%$ for $\alpha \geq 15^{\circ}$, whereas the flat plate shows a higher uncertainty due to lower drag forces with $5 \%$ at $\alpha=0^{\circ}$ and $<1 \%$ for $\alpha \geq 15^{\circ}$.

The dynamic behaviour of the experimental setup is separately validated to obtain the eigenfrequencies and damping characteristics of all its electro-mechanical components. The force transducer itself is measured to have a natural frequency of $5 \mathrm{kHz}$ with an underdamped behaviour with damping ratio of $\zeta=0.47$. The dynamics of all wing model structures are measured, avoiding resonance points for the measured wind/rolling road speed. The spectral content of the rolling road/sting, wind tunnel fan and the attached wings appears remarkably constant for all angles-of-attack and had significantly lower amplitude levels $(\Delta=20 \mathrm{~dB})$ compared with membrane wing induced force and membrane fluctuations. Clear changes in spectra of membrane wing force/moment coefficients with modifying angle-of-attack and heightover-ground show that the setup eigenfrequencies do not significantly influence the results.

\subsection{Deformation measurement}

Stereoscopic Digital-Image-Correlation (DIC) is carried out to measure instantaneous membrane deformations using two high-speed cameras (Phantom V341). The field of view is $1312 \times 1000$ pixels, capturing the entire membrane wing surface. Volumetric calibration is conducted using a calibration plate. The black membranes are sprayed with a white random speckle pattern with $\sim 3$ to 5 speckles per mm ( $\sim 2$ pixels per speckle). The patterns are illuminated with light emitting diodes (LEDs). High-speed images are captured at a sampling-rate of $800 \mathrm{~Hz}$ over a sampling-time of $10 \mathrm{~s}$ for one of the five sample wings from the force/moment measurements. A total of 8000 images are obtained, spanning over 50 cycles of the most energetic modes. The images are captured simultaneously with the aerodynamic loads, allowing correlation of membrane and force dynamics.

Commercial software, LaVision StrainMaster, is used to obtain deformations from the stereo images. A subset size of $89 \times 89$ pixels with a grid step of 15 pixels is chosen, resulting in an 
average spatial resolution of $0.03 c$. The uncertainty of the membrane deformation is estimated to be no worse than $0.1 \%$ of the chord length and is measured by recording two images in still position while comparing the displacement between them. The DIC-technique is widely used in experimental mechanics, allowing sub-pixel accuracy due to grey value interpolation schemes over the interrogation grid (Schreier et al., 2000). The results agree with previous studies, applying DIC on membrane wings (Rojratsirikul et al., 2011; Galvao et al., 2006; Stanford et al., 2014).

Out-of-plane displacements of the membrane $(z / c)$ are referenced with respect to the plane coinciding with the wing's rigid frame. Final results include mean membrane deflections $(\bar{z} / c)$, instantaneous $\left(z^{\prime} / c\right)$ and time-averaged fluctuations and their spectral content $\left(S t_{z}\right)$. A POD analysis is carried out using the instantaneous fluctuations $\left(z^{\prime} / c\right)$ over all 8000 images. The same analysis with half the number of images exhibited the same mode shapes and energy content. Therefore, the POD modes are converged and confirm that the ensemble size is sufficient.

\section{RESULTS: FORCES AND MOMENTS}

\subsection{Average lift and drag of rigid and membrane wings}

Figure 4 presents the influence of various ground heights $h / c$ on the lift $C_{L}$ and $\operatorname{drag} C_{D}$ coefficient of rigid flat plates and membrane wings. Firstly, both wings show lift enhancement for $h / c \leq 1$, which matches previous results for higher (Yun et al., 2010) and lower (Rozhdestvensky, 2006) Reynolds numbers.

At low to moderate angles-of-attack of $0^{\circ} \leq \alpha \leq 15^{\circ}$, membrane wings show a significant benefit of up to $40 \%$ in total lift coefficient $C_{L}$ in comparison to rigid flat plates. The membrane's benefit already exists for the initial unbounded flow environment at $h / c=2$ and can mainly be related to flow induced cambering of the membrane, resulting in lift enhancing pressure modification on the suction-side of the wing, seen in previous rigid cambered wings (Pelletier and Mueller, 2000) and membrane wing studies (Visbal et al., 2009; Rojratsirikul et al., 2010; Shyy et al., 2005; Gordnier and Attar, 2014).

It should be mentioned that the slope $d C_{L} / d h$ (Figure $4 \mathrm{a}$ ) of rigid wings shows for most incidences a decreasing trend with height, allowing favourable stability characteristics in height. In other words, a reduction in height (due to a disturbance) results in a recovery due to gain in lift and vice versa. However, the slope appears slightly positive in the region of wing stall at $17.5^{\circ} \leq \alpha \leq 25^{\circ}$, resulting in instability in this region (loss of height is accompanied with lift loss). Membrane wings (Figure 4b) exhibit a similar unstable slope region in stall, however at higher angles-of-attack of $22.5^{\circ} \leq \alpha \leq 35^{\circ}$ and with significantly stronger positive slope and therefore more distinct unstable behaviour. The stability in height recovers for very close ground clearances of $h / c \leq 0.1$. A previous study (Rhodes and Sayers, 2009) explains the flow physics behind this phenomenon of slope modification within ground-effect using two different effects. The reduction in height into ground-effect $(0.1 \leq h / c \leq 2)$ forces the incoming flow at the leading edge to become parallel to the ground surface. As a result, the effective angle-ofattack is reduced, causing a drop in lift. Further descent into extreme ground-effect of $h / c \leq 0.1$ causes the the ram-pressure below the wing to gain superiority, accompanied with an increase in lift.

The increase in lift within ground-effect comes at a price of higher drag (Figure 4c and Figure 4d). However, the rise in drag is less than the benefit in lift, especially at low angles-of- 


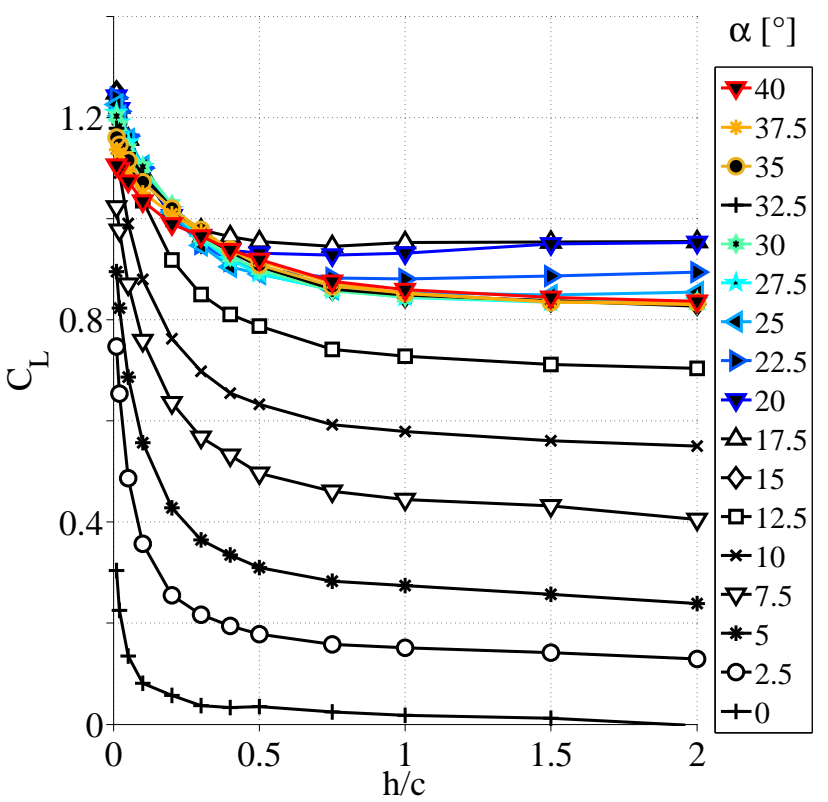

(a) Lift rigid

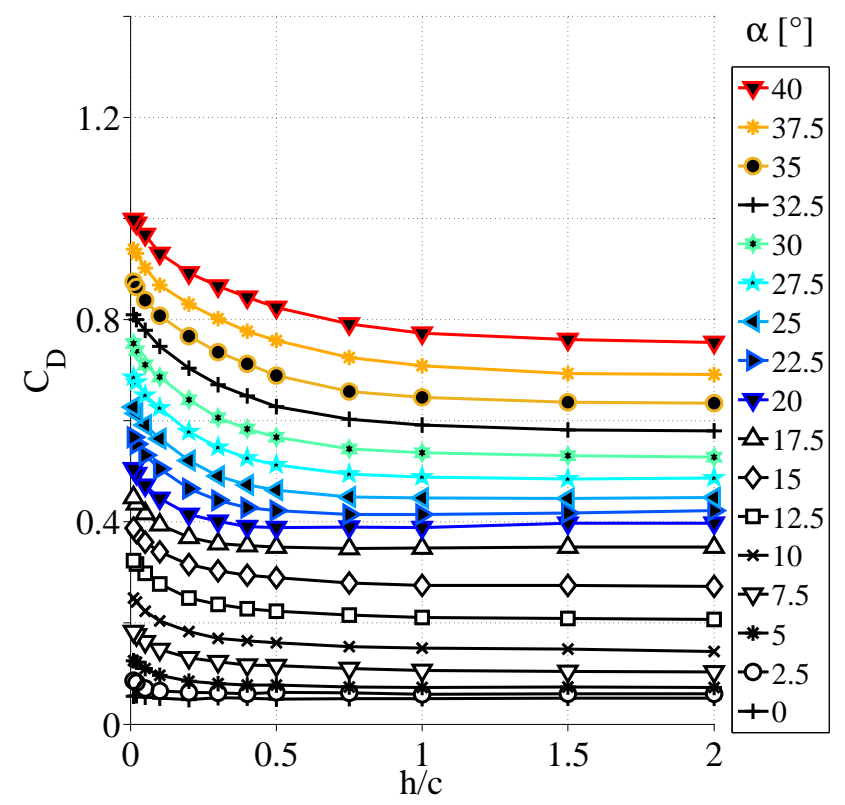

(c) Drag rigid

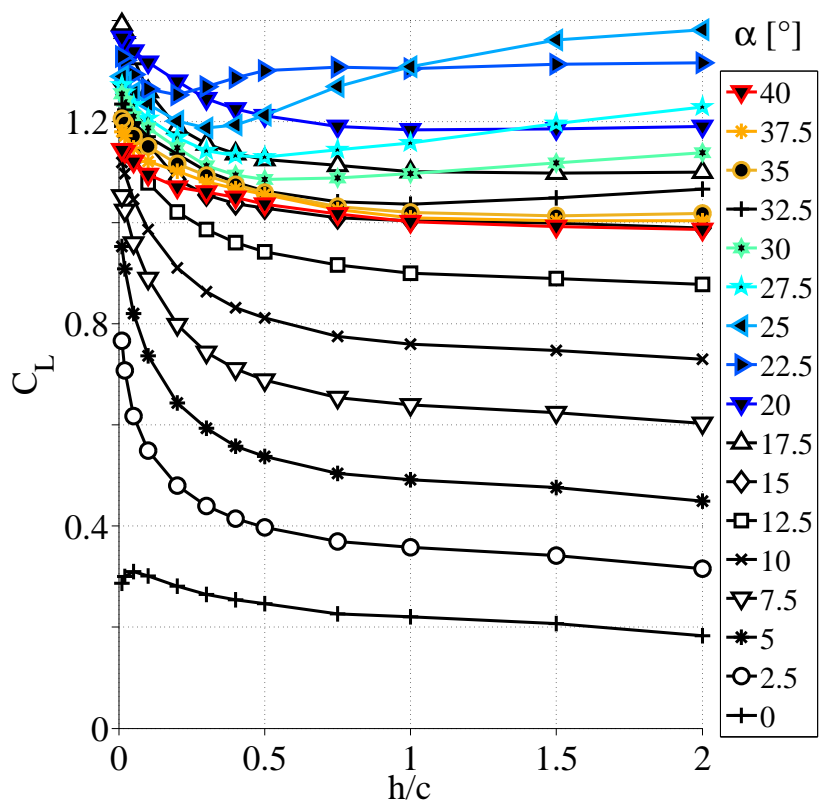

(b) Lift membrane

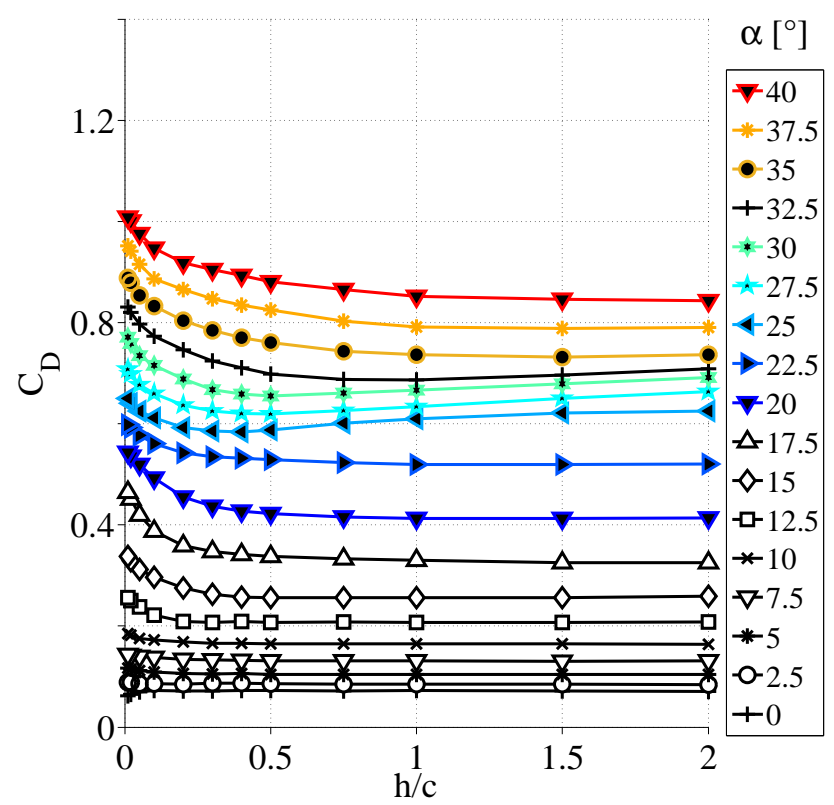

(d) Drag membrane

Fig. 4 Lift and drag coefficient for rigid and membrane wings, changing distance to ground at constant $\alpha$. 
attack of $\alpha \leq 5^{\circ}$ where the drag curve reveals almost no change in drag with decreasing height. The drag of membrane wings for $22.5^{\circ} \leq \alpha \leq 35^{\circ}$ exhibits a decreasing trend with reducing height which correlates to a negative lift-slope (Figure 4b).

\subsection{Lift dynamics of membrane wings}

Figure 5 shows the fluctuation intensity of the lift coefficient $\sigma\left(C_{L}\right)$ (Figure $5 \mathrm{a}$ ) and its power spectral density $P S D\left(C_{L}\right)$ (Figure 5b), changing with angle-of-attack and height above ground. The Strouhal number $S t_{C_{L}}$ in Figure $5 \mathrm{~b}$ represents the frequency $f$ normalised with free-stream velocity $U_{\infty}=8.4 \mathrm{~m} / \mathrm{s}$ and chord length $c=0.1 \mathrm{~m}$. The drop in dominant frequencies in nearstall conditions are labelled and marked in blue.

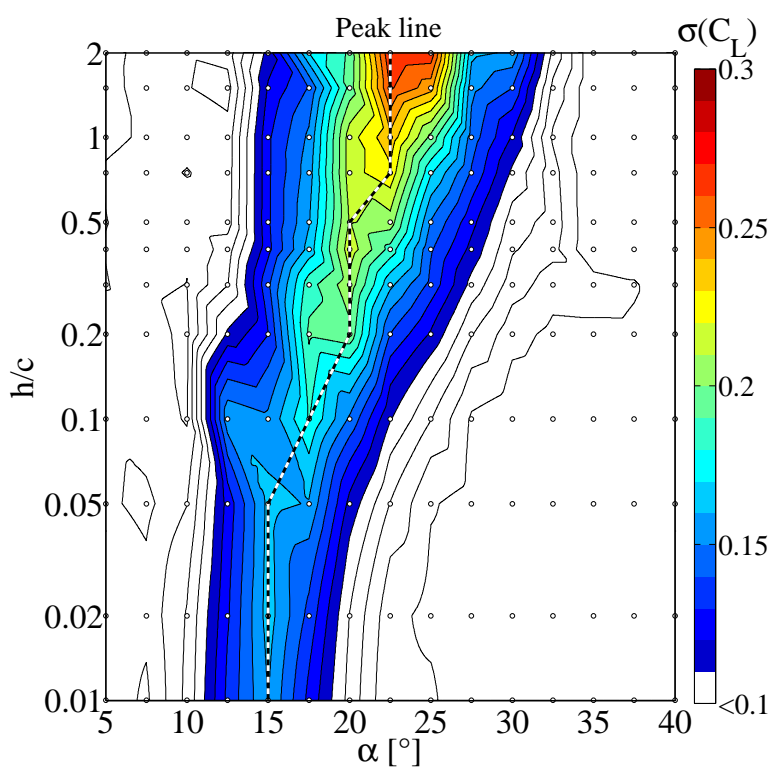

(a) Averaged fluctuation intensity in lift

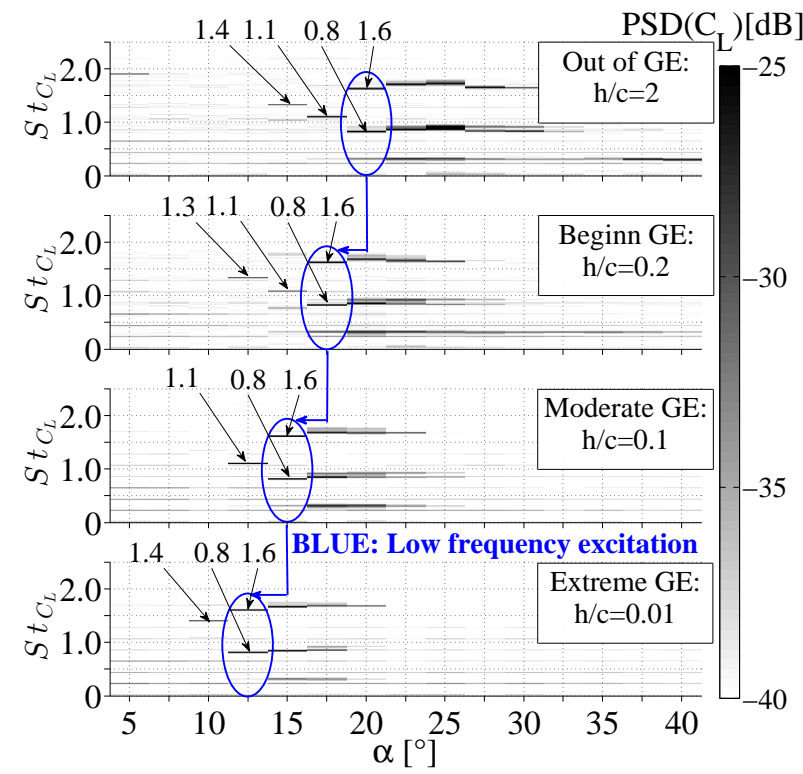

(b) Spectra of lift fluctuation (waterfall)

Fig. 5 Variation of lift fluctuations $(a)$ and its spectral content $(b)$ with angle-of-attack and height above ground

For a given height (Figure 5a), lift fluctuations are found to peak close to wing stall, reaching $\sigma_{\max }=0.3$ at $\alpha=22.5^{\circ}$ in free-flight conditions of $h / c=2$. This intensity halves in strength to $\sigma_{\max }=0.15$ and moves to $\alpha=15^{\circ}$ in close proximity to the ground at $h / c=0.01$ (see dotted line in Figure 5a). This could be due to the weaker pressure fluctuations on the upper side of the wing surface when it is closer to the ground minimising the dynamics of the flow. Additionally, tip-vortices are known to reduce in size and vorticity in ground-effect (Han and Cho, 2005), which may also contribute to a global decrease in flow/structure/force dynamics of membrane wings. Nevertheless, at fixed moderate angles-of-attack, ranging from $5^{\circ} \leq \alpha \leq 15^{\circ}$, membrane wings show an increase in force fluctuations with decreasing ground height, which is perhaps to due to the premature onset of leading edge flow separation, as found in previous studies (Prasad and Damodaran, 2013).

Inspection of the spectral content of lift fluctuations of membrane wings (Figure 5b) reveals that the frequencies at the onset of stall change with ground clearance. Free flight conditions of $h / c=2$ and moderate angles of $10^{\circ} \leq \alpha \leq 15^{\circ}$ exhibit high dominant frequencies of $S t_{C_{L}}=$ 
1.4, typically caused by a high membrane tension and driven by boundary layer perturbations (Rojratsirikul et al., 2009). Increasing the angle-of-attack to $15^{\circ} \leq \alpha \leq 20^{\circ}$ causes a leading edge vortex induced stairs-like drop into lower dominant frequency levels from $S t_{C_{L}}=1.4$ to 1.1 to 0.8 . In the vicinity of stall-angles of $\alpha \geq 20^{\circ}$ and beyond, dominant frequencies in lift remain at $S t_{C_{L}}=0.8$ and its higher harmonics. The fluctuations for the stall region and beyond are most likely driven by the influence of leading-edge-vortex shedding.

Lowering the height from free-flight conditions $(h / c=2)$ to extreme ground-effect $(h / c=$ 0.01) causes the dominant frequencies (blue encircled in Figure 5b) to shift to lower anglesof-attack (a shift of $\Delta \alpha=7.5^{\circ}$ is observed). This might be linked with the reduction in downwash-angle, caused by the close proximity of the ground (Vogt and Barber, 2012). At a fixed angle of $\alpha=15^{\circ}$, membrane wings show a decrease in dominant frequency in lift from $S t_{C_{L}}=1.4$ to 0.8 with a decrease in height. Previous studies on rigid wings in ground-effect observed a reduction in vortex shedding-frequency in the vicinity of the ground, which might explain the modification in lift dynamics due to coupling (Prasad and Damodaran, 2013). However, further flow-membrane-force investigations are necessary for further elaboration.

\subsection{Pitch statics and dynamics of rigid and membrane wings}

The pitching moment is measured about the quarter chord position and its time-averaged value is shown in Figure 6a for rigid-flat plates and membrane wings in free-flight $(h / c=$ $2)$, moderate $(h / c=0.1)$ and extreme $(h / c=0.01)$ ground-effect conditions. Additionally, Figure $6 \mathrm{~b}$ illustrates the fluctuations in pitching moment $\sigma\left(C_{M}\right)$ of the membrane wings for different ground heights.

At a constant height, membrane wings exhibits a pitching moment slope that is near horizontal, likely due to the variable cambering of the membrane with angle-of-attack. This results in an inferior static stability performance of membrane wings compared to rigid wings (Figure 6a) for low angles-of-attack. The absolute value of the pitching moment appears more negative for a membrane wing, a behaviour known to be linked with changes in pressure distribution, and is comparable to previous studies (Bleischwitz et al., 2014).

The reduction in height-over-ground translates the pitch curves down vertically. Earlier flow separation within ground-effect is acting similar to an increase in angle-of-attack in free-flight conditions. Therefore, an typical pitching moment of $C_{M}=-0.1$, measured at a rigid-flat plate in extreme ground-effect at $\alpha=5^{\circ}$, is found again at $\alpha=15^{\circ}$ for free-flight conditions.

The flexibility of the membrane allows flow induced pitching moment fluctuations $\sigma\left(C_{M}\right)$, which change with angle-of-attack and height (Figure 6b). The fluctuations in pitching moment around $\alpha=0^{\circ}$ are large, primarily because the membrane is under very limited flow-induced tension, resulting in large membrane oscillations that directly affect the dynamics of the pitching moment. At $5^{\circ}$, there is a balance between the flow-induced tension and gravity where there is decline in fluctuations with decreasing height above ground. The fluctuations reduce drastically at moderate angles-of-attack due to a gain in aerodynamic loading causing the membrane to stretch and stiffen (Figure 7). This region is also relatively unaffected by leadingedge vortex-shedding, avoiding significant membrane excitation. At angles-of-attack near stall, there is marked increase in fluctuations. This behaviour might happen due to growth influence of leading-edge vortex-shedding, which could excite the membrane and thereby affecting the pitching moment. Finally, variations in pitching moment reduces drastically in post-stall conditions, because the vortices that might shed from the leading-edge are no longer attached to 


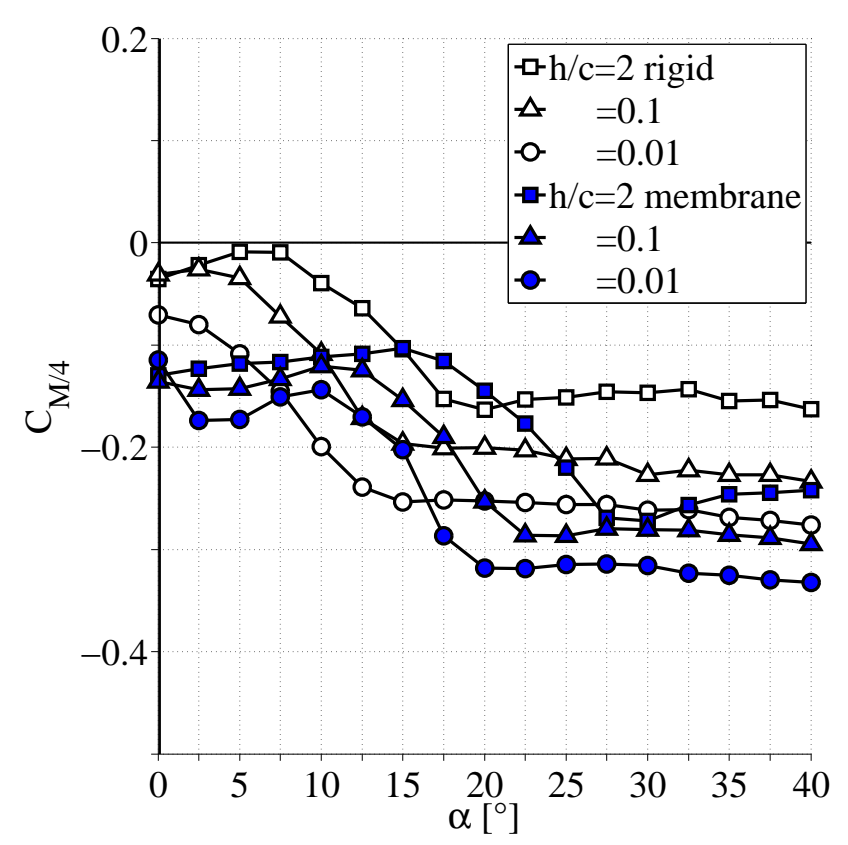

(a) Mean: Rigid + Membrane
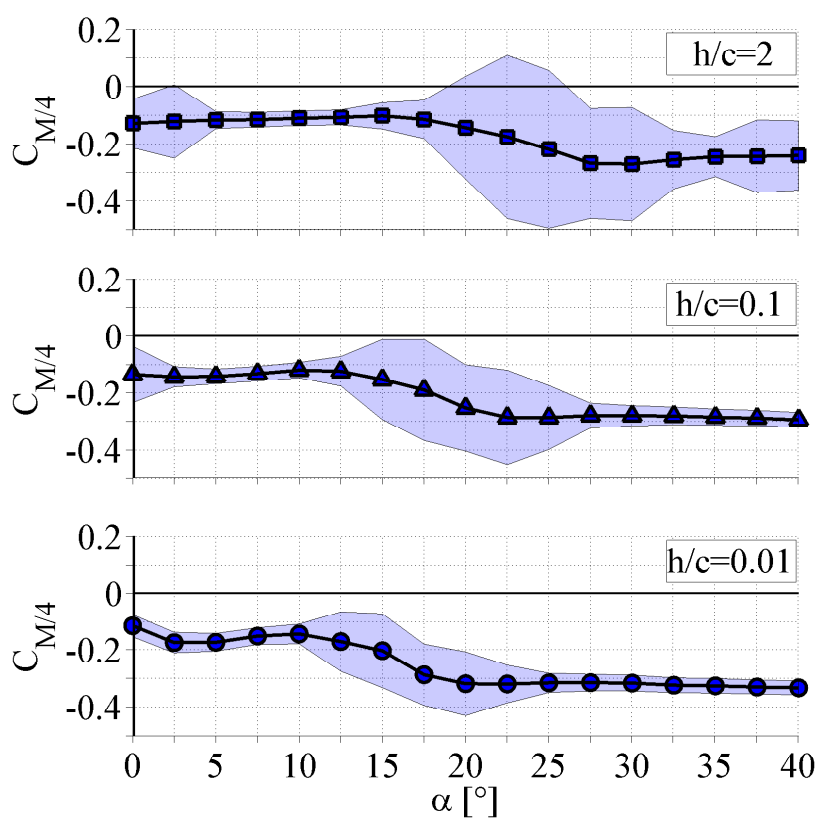

(b) Fluctuation intensity: Membrane

Fig. 6 (a) Time-averaged pitching moment coefficient $C_{M / 4}$ for rigid flat plate and membrane wing in and out of ground-effect. (b) Fluctuations of $\sigma\left(C_{M / 4}\right)$ around the mean is displayed for membrane wings in blue shading.

the membrane surface and therefore do not influence the load distribution on the membrane in a significant way. Further details of flow-membrane interaction, modifying with angle-of-attack can be found in the literature (Gordnier and Attar, 2014; Hu et al., 2008; Tregidgo et al., 2011; Rojratsirikul et al., 2009).

The descent into ground-effect causes the maximum in fluctuations to decrease. This could be due to the fact that the lift generation in ground-effect is dominated by the flow/pressure distribution on the lower wing surface. This could sufficiently reduce the dynamic influence of the upper surface. However, further flow visualisation is required to analyse this effect.

\section{RESULTS: MEMBRANE DEFORMATIONS}

\subsection{Statistics}

Average membrane-deformations $\bar{z}$ and fluctuations $\sigma(z)$ are obtained by averaging the instantaneous surface results $z(x, y, t)$ over the entire time series (Figure 7a). The flexibility of the membrane allows shape adaption with changes in angles-of-attack and height. Figure 7b shows the cambering of the membrane at mid-span for $\alpha=5^{\circ}$ and $15^{\circ}$. A reduction in height causes the membrane to increase its mean camber due to gain in positive pressure below the wing surface. The lower angle of $\alpha=5^{\circ}$ shows a higher relative increase in camber when the wing is closer to the ground compared to the higher angle of $\alpha=15^{\circ}$. Lowering the height at $\alpha=5^{\circ}$ also causes the chord position of maximum camber to move towards the leading-edge, which is similar to the response to an increase in angle-of-attack.

Figure 8 shows the variation in maximum membrane mean deformation $\bar{z}_{\text {max }} / c$ and fluctu- 

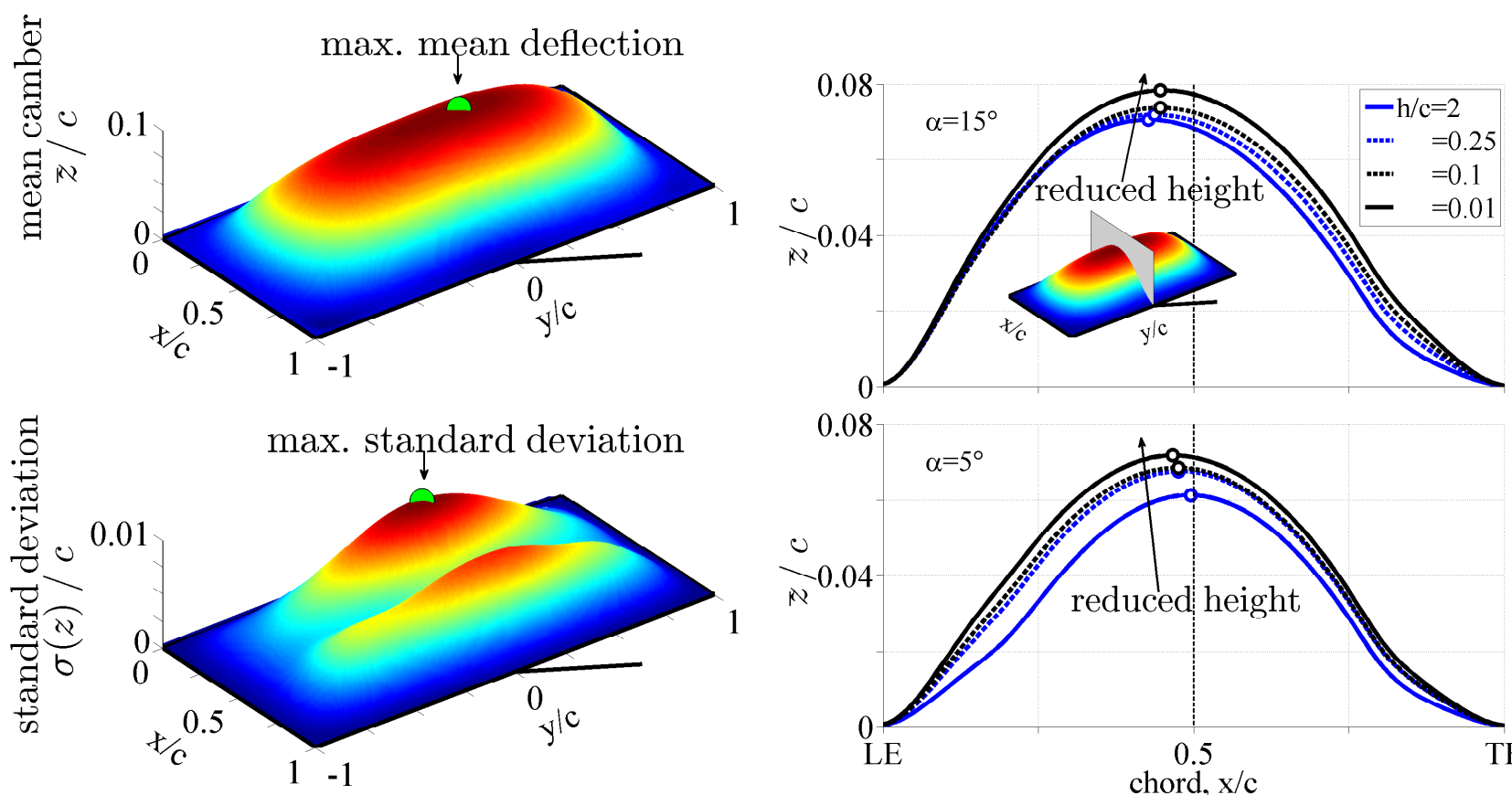

(a) Mean and std of membrane surface

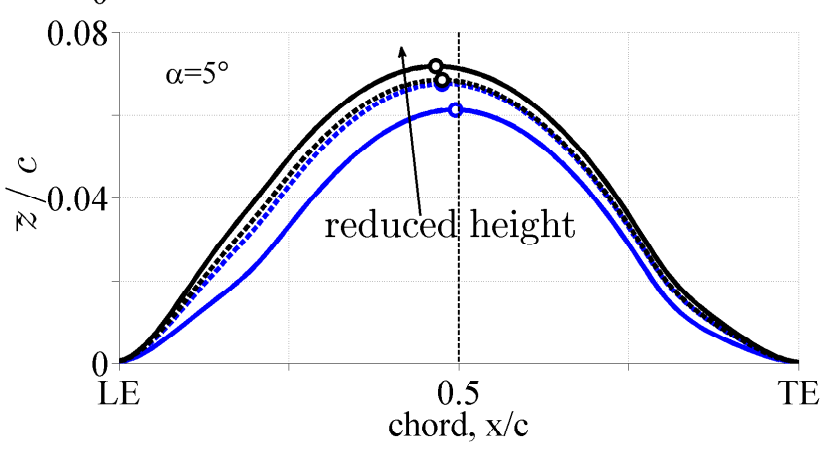

(b) Cross-section modification with height

Fig. 7 (a) Exemplary time-averaged membrane mean surface deflection and fluctuation intensity $\left(h / c=2, \alpha=25^{\circ}\right)$. (b) Cross-sections at mid-span for mean camber modifying with $\alpha$ and $h / c$. Note the steaper incline in camber within ground-effect for the lower angle-of-attack.

ation intensity $\sigma(z) / c$ of the membrane surface, varying with ground clearance and angle-ofattack. The DIC-technique ensures a maximum deformation uncertainty of $\mathrm{z} / \mathrm{c}=0.001(0.1 \%$ of chord, equal $0.1 \mathrm{~mm}$ ). Membrane wings at $h / c=2$ exhibit a steady increase in maximum camber with angle-of-attack reaching $0.08 \mathrm{c}$ at $25^{\circ}$ (Figure 8 ). This result compares well within previous findings of a wing of similar aeroelastic properties (Rojratsirikul et al., 2011). At these free-flight conditions, the maximum camber deflection is shown to peak with $\alpha=25^{\circ}$ at the same angle-of-attack found for maximum lifting capability (see Figure 4b). The membrane camber is found to decrease in post-stall conditions at $h / c=2$, related to the loss in lift generation.

The vicinity of the ground forces the membrane to modify its camber in a way that is similar to changes in angle-of-attack in free-flight conditions (Figure 8). Low angles-of-attack $\left(\alpha \approx 5^{\circ}\right)$ show the highest increase in maximum camber $(16 \%)$ between free-flight conditions at $h / c=2$ and extreme ground-effect at $h / c=0.01$. This result can also be correlated with earlier findings of predominant lift increase at low incidences (Figure $4 \mathrm{~b}$ ). Ground-effect was found to induce earlier stall, resulting in a drop in membrane camber in extreme ground-effect of $h / c \leq 0.1$ at $\alpha=20^{\circ}$ and $22.5^{\circ}$ (Figure 8). At even higher incidences of $\alpha=35^{\circ}$, the membrane camber increases steadily with decreasing distance to the ground, suggesting predominant pressure build up on the lower surface of the wing and further lift enhancement despite a large stagnantflow zone on the upper side of the wing. Ultimately, changing angle-of-attack in ground-effect causes smoother and more steady modifications in membrane displacement when compared to free-flight. Further flow visualisation should be carried out to correlate membrane with flow modifications. 


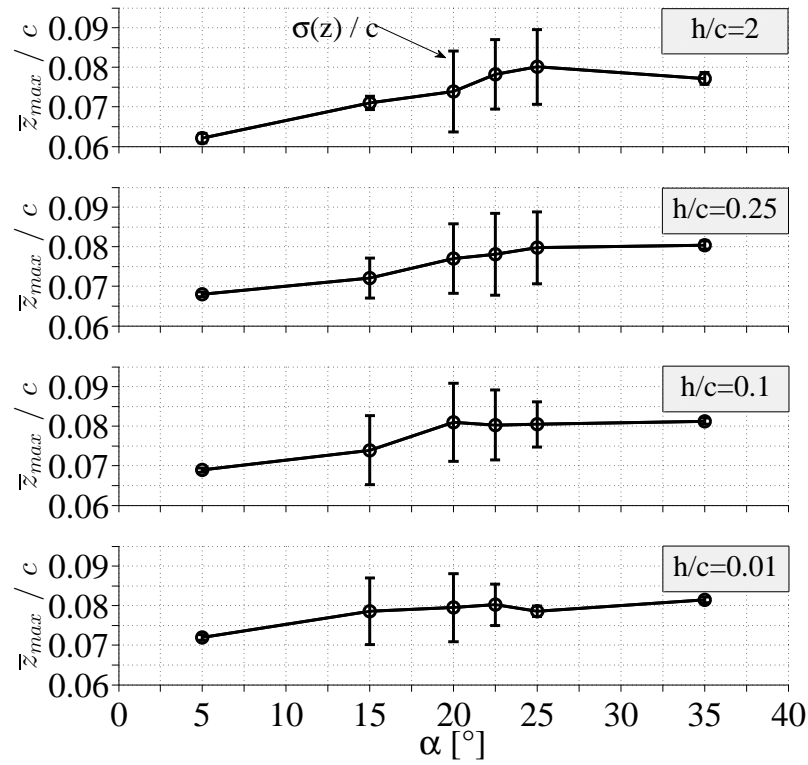

Fig. 8 Variation of membrane maximum mean deflection and maximum standard deviation with angle-of-attack for four heights-over-ground.

The predominance of leading-edge vortex-shedding is known to significantly affect the amplitude of camber oscillations close to stall angles (Gordnier and Attar, 2014). This behaviour can clearly be seen in free-flight conditions of $h / c=2$ with a stall-angle of $\alpha=25^{\circ}$ (Figure 8, top), where the fluctuation intensity $\sigma(z) / c$ reaches nearly $10 \%$ of mean value. In comparison, low angles-of-attack around $\alpha \sim 5^{\circ}$ do not exhibit any significant membrane vibrations at all, not even in ground-effect. This might be a result of a mostly attached flow without any large leading-edge vortex-shedding which could trigger large oscillations in the membrane. However, an increase in incidence to $\alpha \sim 15^{\circ}$ shows a measurable impact in growing membrane fluctuations, gaining even more strength at lower ground clearances. It is suggested that this may be linked with the growing influence of the leading-edge vortex and its shedding, gaining strength with higher incidences or lower ground clearances. For very high angles-of-attack $\left(\alpha \geq 25^{\circ}\right)$, a steep drop in membrane fluctuations is observed when going from free-flight conditions into ground-effect (Figure 8). This phenomenon appears to be similar to that observed when incidence angles go from the stall to the post-stall regime at a constant height. This suggests that the dynamics of membrane wings in ground-effect might be predominately driven by flow modifications that are similar to those seen for angle-of-attack changes at a constant height (Bleischwitz et al., 2015b).

\subsection{Decomposition of membrane dynamics}

Proper-Orthogonal-Decomposition (POD) has previously successfully been applied to the flow field around membrane wings (Schmit et al., 2003; Lian et al., 2003) as well as membrane dynamics (Stanford et al., 2010; Buoso and Palacios, 2015; Shi et al., 2013). The complexity of membrane-vibrations requires a technique which is capable to identify and quantify twodimensional spatial structures in membrane-oscillations. The use of POD allows to capture those most energetic structural vibration mode shapes directly from time resolved image data 
(Han and Feeny, 2003). The POD modes of the membrane deformations are identified using the snapshot method. Details of the method is excluded from the paper and the reader is referred to other sources for details (Lumley, 1967; Sirovich, 1987; Pinnau, 2008; Kostas et al., 2002).

Figure 9a show the first eight POD mode shapes of membrane vibrations (excluded mean). These modes are in order of decreasing energy content as shown in the figure. Three heights $h / c$ $=[2,0.25,0.1]$ at a fixed angle-of-attack of $\alpha=15^{\circ}$ are selected for brevity. The time-series of POD mode weights $\left(a_{i}\right)$ are computed by projected every instantaneous membrane deformation in to their respective POD modes and this data is used to compute the frequency spectra of the mode weights.

In free-flight conditions $(h / c=2)$, the vibration behaviour is mostly chordwise, with $29 \%$ of all energy captured in the $1^{\text {st }}$ mode $a_{1}$ (four chordwise extrema), $16 \%$ in the $2^{\text {nd }}$ mode $\left(a_{2}\right.$, three chordwise extrema) and nearly $11 \%$ in the $3^{\text {rd }}$ mode ( $a_{3}$, two chordwise extrema). Spanwise modes are visible but with very low energy content $(<4 \%)$. This shows that the energy is distributed over these few modes. This energy distribution is consistent with previous membrane wing studies, where the technique of sinusoidal decomposition was applied at a fixed span location (Bleischwitz et al., 2015b). The first POD coefficient $a_{1}$ shows a dominant membrane vibration frequency of $S t_{a_{1}}=1.3$ (Figure $9 \mathrm{~b}$ ) which is comparable to the frequency found in the lift coefficient (Figure 5b). Higher modal coefficients, $a_{2}, a_{3}, a_{4}$, despite their lower spatial order, show strong excitation at the same frequency of $S t_{a_{1}}=1.3$. This suggests that there is strong coupling between these mode shapes and that the spatial structures are essentially sub-harmonics of the fundamental mode shape.

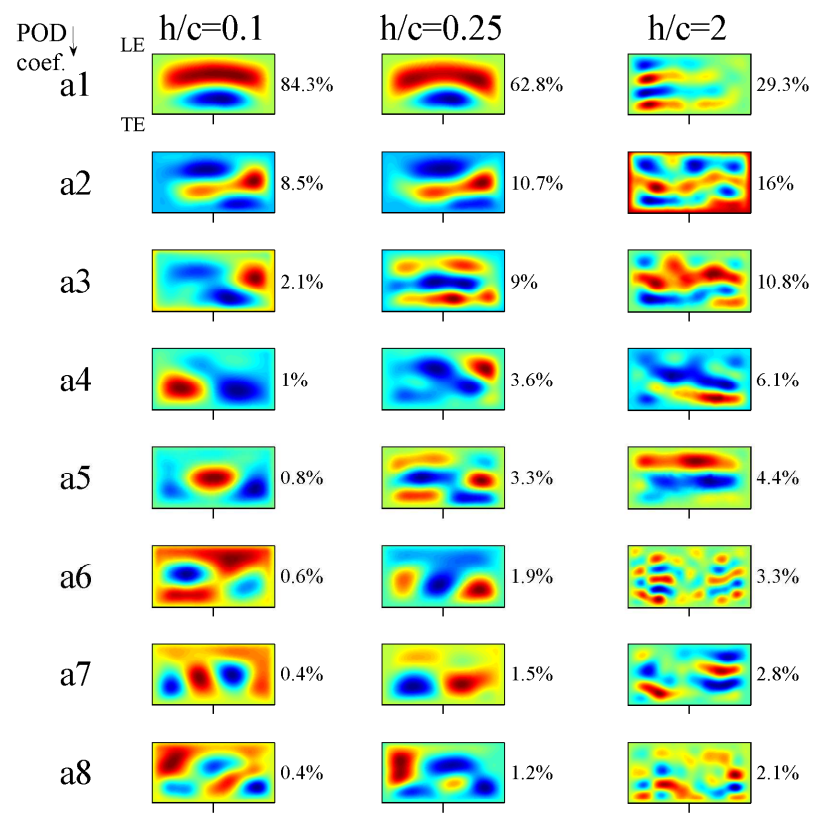

(a) First eight POD-modes with energy content

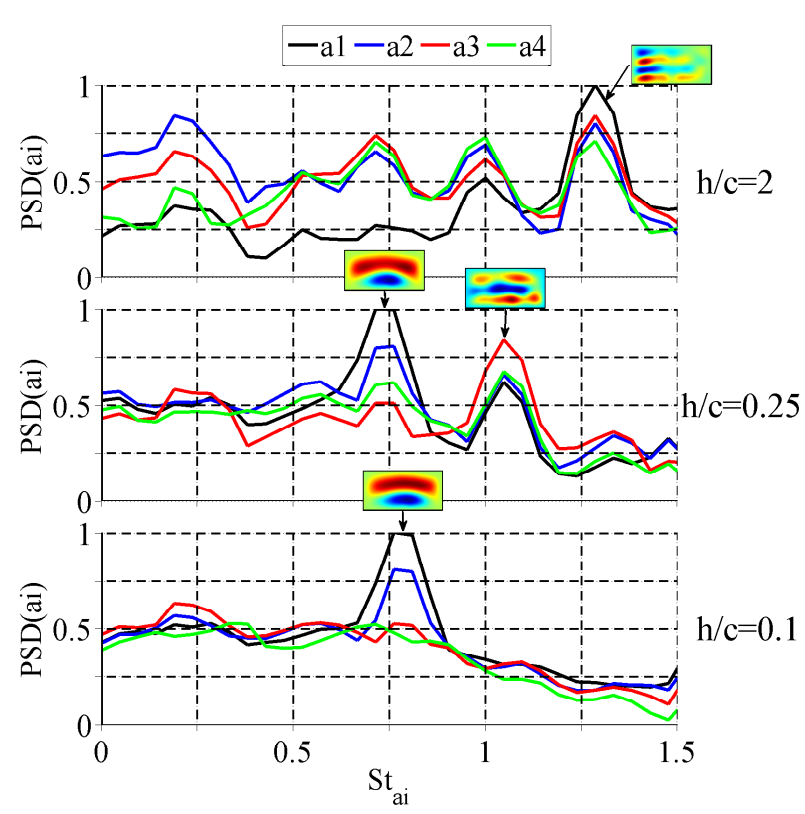

(b) Spectral energy in first four POD coefficients

Fig. 9 POD analysis on membrane fluctuations, showing spatial $(a)$ and spectral $(b)$ contribution of POD modes at a fixed incidence of $\alpha=15^{\circ}$ for different heights above ground.

Membrane vibrations are found to be strongly affected by the reduction in height. A reduction in height from $h / c=2$ to $h / c=0.25$ causes the most dominant mode shapes to change from four local extremes along the chord to just two. This is perhaps due to the increasing influence of vortex-shedding when the membrane is in ground-effect. In addition, the individual 
energy content appears less evenly distributed over different POD-coefficients, accumulating almost $63 \%$ in the first mode, $11 \%$ in the $2^{\text {nd }}$ mode and $9 \%$ in the $3^{\text {rd }}$ mode. Note that the mode shapes at $h / c=0.25$ is different from those in $h / c=2$. The increased vibration amplitude of the membrane allows us to clearly identify spanwise modes $\left(\geq a_{4}\right)$ of low energy levels $(<4 \%)$. Examining the spectral content of the first POD shapes shows that at this height above ground $(h / c=0.25)$, two different frequencies appear to be dominant $\left(S t_{a 1}=0.8\right.$ and $\left.S t_{a 3}=1.1\right)$. These frequencies are consistent with the frequencies observed in the force dynamics (Figure 5b).

A further reduction in altitude to $h / c=0.1$ supports the trend of accumulation of energy in fewer modes (in this case $85 \%$ and $9 \%$ in the first two modes, respectively). The most dominant mode again has two chordwise nodes, representative for a flow in near-stall conditions (Rojratsirikul et al., 2011). The spanwise modes carry very little energy $(\leq 1 \%$ to the total vibration energy in the membrane). The POD coefficient $a_{6}$ at $h / c=0.1$ illustrates a special case of a diagonal structure with a low energy content $<1 \%$. The diagonal shape could be a result of alternating tip vortex roll up or simply non-uniform boundary condition in membrane attachment. Further investigation is required to explain the source of these observed PODmodes.

Overall, the POD analysis has shown that the first two modes are sufficient to capture $90 \%$ of membrane deformation for low values of $h / c$ at moderate angles-of-attack of $\alpha=15^{\circ}$, where the flow-dynamics are most likely be dominated by strong leading-edge vortices. In contrast, eight modes are required to capture $75 \%$ of membrane deformation at the same incidence in free-flight conditions, where membrane dynamics are more likely be driven by boundary layer perturbations. Ultimately, membrane mode shapes are shown to be different in ground-effect and the differences between ground-effect and free flight at a fixed angle-of-attack are similar to differences observed across angles-of-attack in free-flight (Bleischwitz et al., 2015b).

\section{RESULTS: AERODYNAMIC COUPLING}

\subsection{Coupling between membrane motion and aerodynamic forces}

Figure 10a shows a representative time-series of lift $C_{L}(t), \operatorname{drag} C_{D}(t)$ and membrane vibrations $z^{\prime}(t)$ for heights $h / c=[2,0.25,0.1]$ at an angle-of-attack of $\alpha=15^{\circ}$. The membrane oscillation $z^{\prime}(t)$ is tracked on the membrane surface at the point of maximum vibration intensity. The location of this point changes with height-over-ground due to mode shape modifications, but is always located at the peak closest to the trailing-edge. The normalised in-plane coordinates Figure 7 of that measurement point for the height $h / c=[2,0.25,0.1]$ are given as $[(0.86$ $,-0.49),(0.78,0.14),(0.77,0.14)]$ and are also illustrated within the first instantaneous membrane surface of Figure 10a. Smoothed lines in red (drag) and blue (lift) show downsampled (with a low-pass filter) original force results (gray) to match the sampling frequency of membrane deformations. Contours of time instant membrane fluctuations $z^{\prime}(x, y, t)$ are illustrated at three specific time steps of the given time series. Finally, the ground-effect case $h / c=0.1$ shows additionally green vertical shadow regions, representing an instantaneous gain in aerodynamic efficiency $C_{L}(t) / C_{D}(t)$ over its time-averaged mean value.

The illustrated cases (Figure 10a) allow detailed examination of modal information and their instantaneous contribution to lift, drag and range efficiency. The strong ground-effect case at $h / c=0.1$ shows a very clear low frequency oscillation of two alternating chordwise 

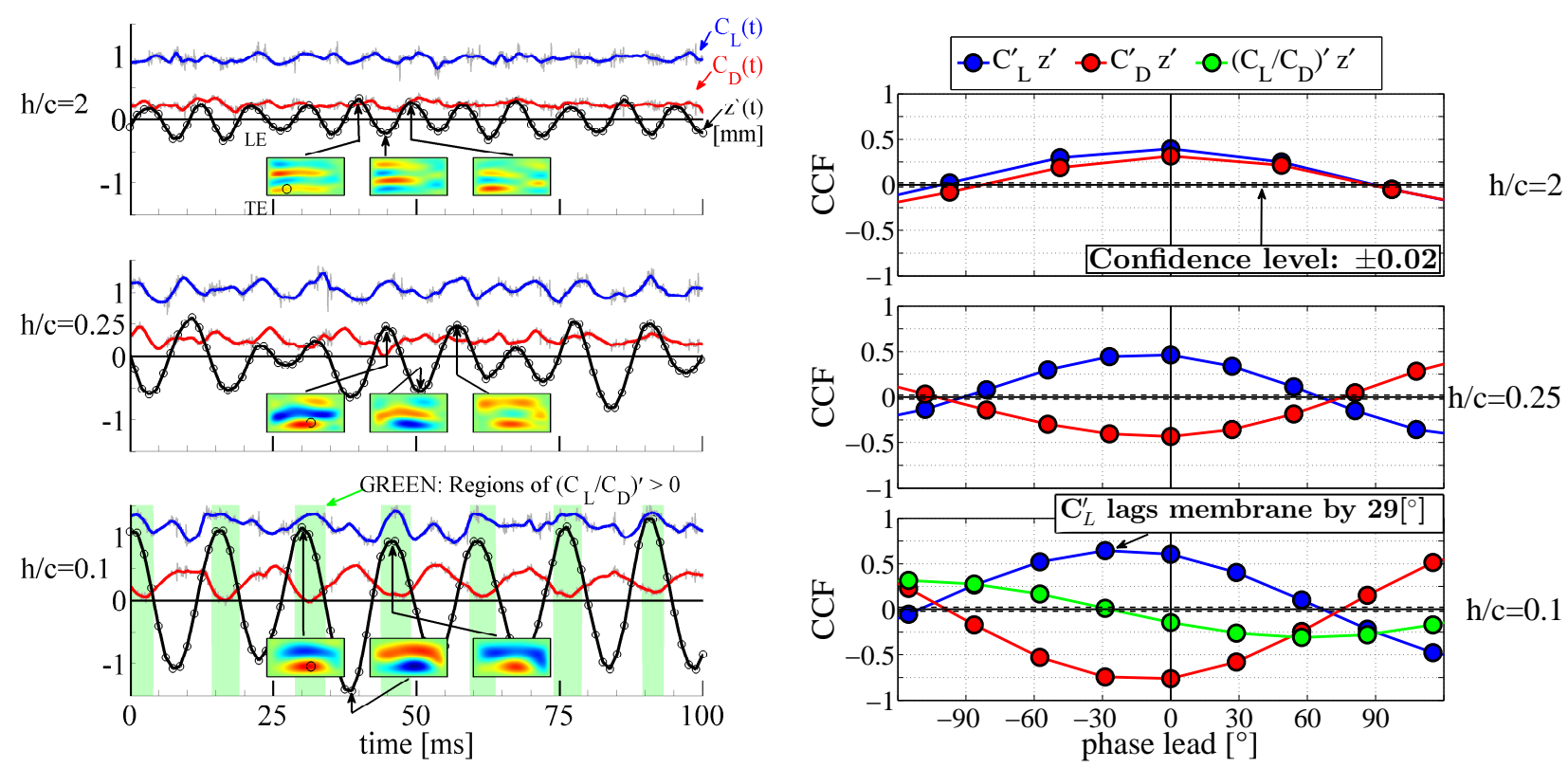

(a)

(b)

Fig. $10(a)$ Time series of $C_{L}, C_{D}$ and instantaneous membrane deformation $\left(z^{\prime}\right)$ for $\alpha=15^{\circ}$ at three different heights over ground. (b) Cross correlation between force coefficients and membrane deformation.

motions in the membrane which suggest considerable influence of strong leading-edge vortex and near-stall flow conditions. It is found that the instantaneous maximum lift is produced when the membrane has a negative fluctuation close to the leading edge (blue in membrane surface is a negative fluctuation relative to the average deformation) and a positive one near the trailing edge (red). In other words, positive increase in lift is associated with an instantaneous rearward location of maximum positive camber. This behaviour might indicate a rolled-up vortex-structure convecting towards the trailing-edge and in the process inducing the membrane deformation, which in turn affects the lift fluctuation. The drag is reduced within this region and can even become negative, producing thrust rather than drag (not shown). As a result, the aerodynamic efficiency (green vertical shading in Figure 10a is found to peak within these periods. A recent study on flapping membrane wings (Jaworski and Gordnier, 2012) provides an explanation for this phenomenon, suggesting that instantaneous pressure distribution focussed on the leading edge allows for a forward tilted (thrust producing) resultant force. A similar mechanism might be at play in the above-mentioned scenario.

A direct comparison of the normalised cross-correlation function (CCF) between forces $\left(C_{L}^{\prime}, C_{D}^{\prime}\right)$ and membrane $\left(z^{\prime}\right)$ fluctuations reveals a nearly instantaneous response in forces to changes in membrane deformations (Figure 10b). The illustrated phase period $\phi$ in $\left[^{\circ}\right]$ is relative to the dominant vibration frequency of the membrane, which is measured as [108, 60, 64$] \mathrm{Hz}$ for $h / c=[2,0.25,0.1]$. For the highly energetic vibrations in ground-effect $(h / c=0.1)$, the lift and drag fluctuations are found to correlate by $\mathrm{CCF}=+0.70$ and -0.75 with a maximum phase lag to the membrane by $29^{\circ}$ (lift=positive correlated, drag=negative correlated). The fluctuation in the $\left(C_{L} / C_{D}\right)^{\prime}$-ratio is found to lead the membrane fluctuation by $58^{\circ}$ (negative correlated). However, the correlation value is low and therefore has to be seen with care.

For $h / c=0.25$ and $h / c=2$, membrane deformations (Figure 10a) are coupled with higher 
frequencies and lower vibration-amplitudes. The correlation between lift, drag and membranefluctuations remains without significant lag but correlation reduces to 0.4 (Figure 10b). However, regardless of height above ground, the fundamental similarity across all three cases is that a high lift is associated with a positive fluctuation in deformation closer to the trailing edge and that the effect of membrane deformation on the forces is almost instantaneous.

\subsection{Coupling between membrane motion and pitching moment}

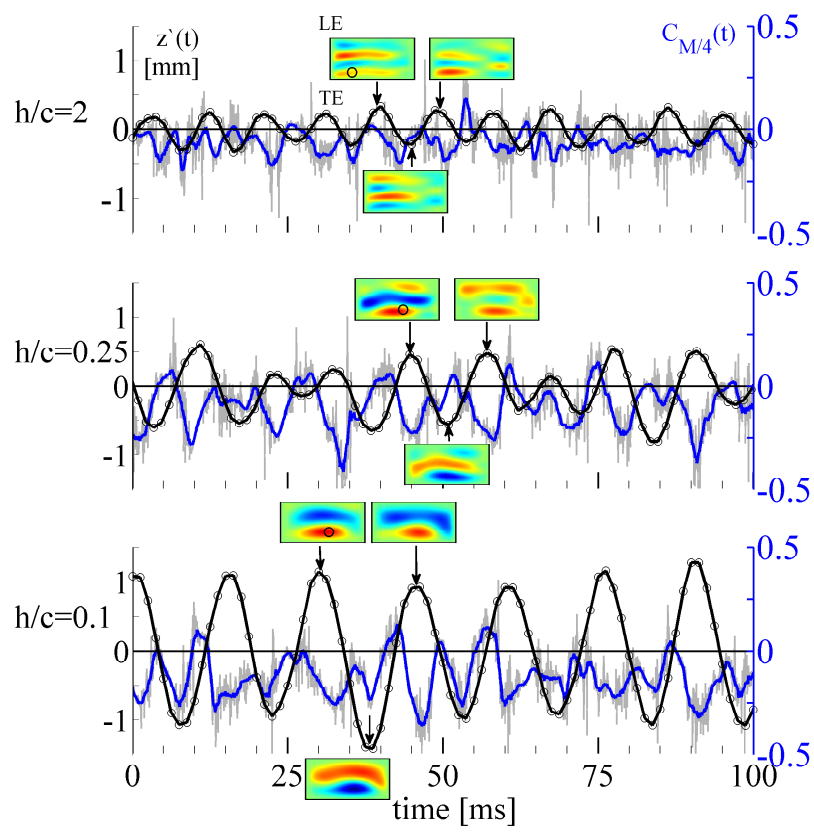

(a)
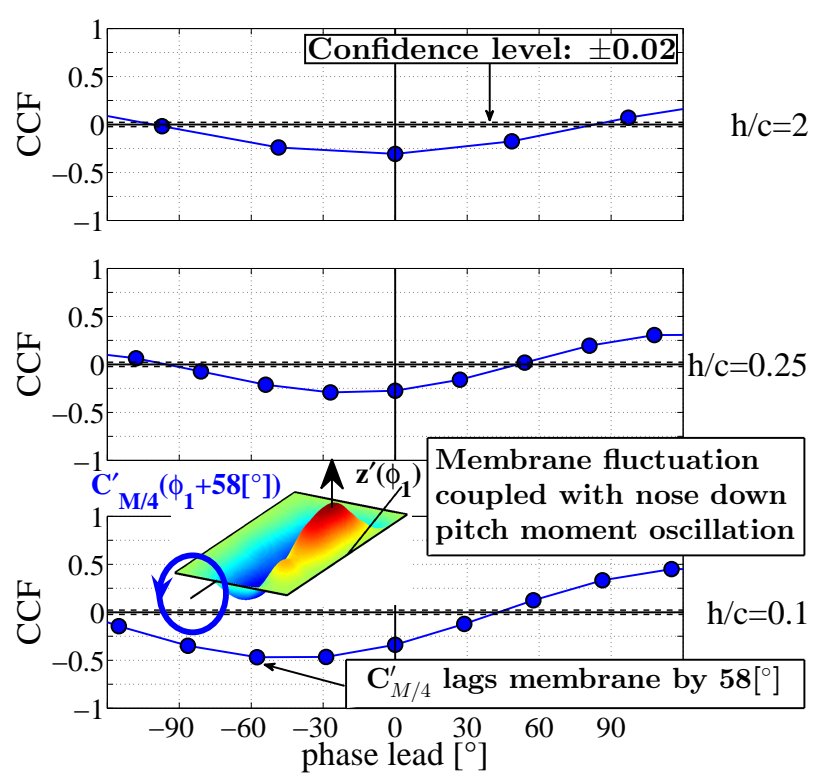

(b)

Fig. 11 (a) Time series of $C_{M / 4}$ and instantaneous membrane deformation $\left(z^{\prime}\right)$ for $\alpha=15^{\circ}$ at three different heights over ground. (b) Cross correlation between $C_{M / 4}$ and membrane deformation.

Figure 11 shows the correlation between the pitch-moment and membrane deformations. Figure 11a shows a time-series of the pitching moment $C_{M / 4}(t)$ (blue-line) and membrane vibrations $z^{\prime}(t)$ (black-line) for three different heights-over-ground $(h / c=[2,0.25,0.1])$ at an angle-of-attack of $\alpha=15^{\circ}$. Figure 11b shows the cross-correlation between two time-series shown in Figure 11a.

At $h / c=0.1$, the normalised cross-correlation value of -0.5 indicates that there is a relatively good match between membrane and pitching moment signal (Figure 11b). The nose-down pitching moment $C_{M / 4}$ lags the trailing-edge orientated positive membrane deflection $+z^{\prime}$ by 58 ${ }^{\circ}$ (as shown in the 3D-surface sketch in Figure 11b). For higher values of $h / c$, the correlation value reduces to -0.3 , indicating a weaker but still negative correlation between membrane deformation and pitching moment. The lag also seems to reduce, becoming closer to zero with increasing $h / c$. However, the measurement uncertainty plays a role in this trend and the data for higher $h / c$, where the membrane vibrations are not large, is critical. In general, flow/membrane induced pitch oscillations can play a major role for coupling and lock-in effects with roll oscillations of low aspect ratio MAV systems as discussed in a recent study(Tregidgo et al., 2012). 


\section{CONCLUSION}

Moderate Reynolds number wind tunnel experiments were conducted using rectangular rigid and membrane wings in the vicinity of the ground. Time-resolved forces/moments and membranedynamics were captured simultaneously, enabling identification of relationship between instantaneous membrane deformations and aerodynamic coefficients.

Both flat-plates and membrane wings show superior lift generation in ground-effect, while maintaining low drag at low to moderate angles-of-attack of $2.5^{\circ} \leq \alpha \leq 7.5^{\circ}$. At higher anglesof-attack, membrane wings benefit from their capability of passive cambering, allowing longer flow attachment and therefore delayed increase in drag. This behaviour becomes an advantage when they are used in ground-effect, where the descent in height is linked with a modification of incoming flow angle, causing generally earlier leading edge flow separation, accompanied with drag increase.

The dynamics of the membrane deformation are key to understanding the unsteadiness in lift/drag/pitch performance. Ground-effect was found to modify membrane dynamics in a way that is similar to the modifications observed for increasing angle-of-attack in free-flight conditions. Ground-effect is seen to force premature flow separation, accompanied with an early onset of leading-edge vortex shedding. As a result, highly energetic membrane mode shapes of low order are excited for decreasing heights above ground.

POD analysis on membrane oscillations allows grouping of major and minor vibration shapes and their energetic contribution. In the vicinity of stall conditions, most of the membrane vibration energy is concentrated in the first two POD modes $(>90 \%)$ of strongly chordwise oriented membrane fluctuations. At large $h / c$, lower energetic (boundary layer driven) membrane vibrations are found with an energy content spread over a larger range of POD modes of more similar energy content. For these conditions, eight POD-coefficients are required to capture $75 \%$ of the total deformations.

Highly energetic and dynamic oscillations, seen for membrane wings in near stall flow conditions, are found to be well correlated to lift, drag and pitching moment modifications. Peaks in forces/moments are found to appear slightly delayed to membrane fluctuation maxima. An exemplary instantaneous rearward location of maximum camber is found to gain lift and correlates to a nose down pitching moment. At the same time, drag is reduced which helps to increase the aerodynamic efficiency.

The results are crucial for future flow control attempts, where time-dependent membrane relaxation could be applied to suppress or enhance individual spatial modes for performance/flow modifications. Furthermore, the study of membrane wings in ground-effect could be an option for the development of long range MAVs, exploiting the vicinity of the ground.

\section{Acknowledgements}

We gratefully acknowledge the support from the Engineering and Physical Sciences Research Council (EPSRC, grant EP/J001465/1) and the European Office of Aerospace Research and Development (EOARD, grant FA8655-12-1-2046). Dr R. de Kat is supported by a Leverhulme Trust Early Career Fellowship. All data supporting this study are openly available from the University of Southampton repository at http://dx.doi.org/10.5258/SOTON/388748 . 


\section{References}

Ahmed, M. and Sharma, S. (2005). An investigation on the aerodynamics of a symmetrical airfoil in ground effect. Experimental Thermal and Fluid Science, 29(6):633-647.

Arbos-Torrent, S., Palacios, R., and Ganapathisubramani, B. (2013). Leading- and TrailingEdge Effects on the Aeromechanics of Membrane Aerofoils. Journal of Fluids and Structures, 38:107-126.

Barlow, J. B., Rae, W. H., and Pope, A. (1999). Low-speed wind tunnel testing. Jhon Wiley \& Sons, Canada.

Bleischwitz, R., de Kat, R., and Ganapathisubramani, B. (2014). Effects of aspect ratio on fluidstructure interactions in membrane wings. In 52nd AIAA Aerospace Science and Technology Forum and Exposition, National Harbor, Maryland.

Bleischwitz, R., de Kat, R., and Ganapathisubramani, B. (2015a). Aeromechanics of Membrane Wings in Ground-Effect. In 45th AIAA Fluid Dynamics Conference, pages 1-28, Dallas, Texas.

Bleischwitz, R., de Kat, R., and Ganapathisubramani, B. (2015b). Aspect-Ratio Effects on Aeromechanics of Membrane Wings at Moderate Reynolds Numbers. AIAA Journal, $53(3): 780-788$.

Buoso, S. and Palacios, R. (2015). Electro-aeromechanical modelling of actuated membrane wings. Journal of Fluids and Structures, 58:188-202.

Carter, A. (1961). Effect of Ground Proximity on the Aerodynamic Characteristics of AspectRatio-1 Airfoils With and With-out End Plates. NASA technical note, 970.

Curet, O. M., Carrere, A., Waldman, R., and Breuer, K. S. (2014). Aerodynamic Characterization of a Wing Membrane with Variable Compliance. AIAA Journal, 52(8):1749-1756.

Diasinos, S., Barber, T. J., and Doig, G. (2012). Influence of wing span on the aerodynamics of wings in ground effect. Proceedings of the Institution of Mechanical Engineers, Part G: Journal of Aerospace Engineering, 227(3):569-573.

Galvao, R., Israeli, E., Song, A., Tian, X., Bishop, K., Swartz, S., and Breuer, K. S. (2006). The Aerodynamics of Compliant Membrane Wings Modelled on Mammalian Flight Mechanics. In 36th AIAA Fluid Dynamics Conference and Exhibit, San Francisco, California.

Gordnier, R. E. and Attar, P. J. (2014). Impact of flexibility on the aerodynamics of an aspect ratio two membrane wing. Journal of Fluids and Structures, 45:138-152.

Han, C. and Cho, J. (2005). Unsteady Trailing Vortex Evolution Behind a Wing in Ground Effect. Journal of Aircraft, 42(2):429-434.

Han, S. and Feeny, B. (2003). Application of Proper Orthogonal Decomposition To Structural Vibration Analysis. Mechanical Systems and Signal Processing, 17(5):989-1001. 
Hays, M. R., Morton, J., Dickinson, B., Chakravarty, U. K., and Oates, W. S. (2012). Aerodynamic control of micro air vehicle wings using electroactive membranes. Journal of Intelligent Material Systems and Structures.

Hsiun, C.-m. and Ghent, C. (1996). Aerodynamic Characteristics of a Two-Dimensional Airfoil with Ground Effect. Journal of Aircraft, 33(2):386-392.

Hu, H., Tamai, M., and Murphy, T. (2008). Flexible-Membrane Airfoils at Low Reynolds Numbers. Journal of Aircraft, 45(5):1767-1778.

Ifju, P. G., Jenkins, D. A., Ettinger, S., Lian, Y., and Shyy, W. (2002). Flexible-Wing-Based Micro Air Vehicles. In 40th AIAA Aerospace Sciences Meeting and Exhibition, number 0705, Reno,NV.

Jaworski, J. W. and Gordnier, R. E. (2012). High-order simulations of low Reynolds number membrane airfoils under prescribed motion. Journal of Fluids and Structures, 31(1):49-66.

Kostas, J., Soria, J., and Chong, M. (2002). Particle image velocimetry measurements of a backward-facing step flow. Experiments in Fluids, 33(6):838-853.

Lee, H. J., Kang, B. J., Park, J. H., Lee, C. M., Kang, K. J., and Kang, C. G. (2011). Development of Hoverwing Type WIG Craft WSH-500. In OCEANS, Yeosu. IEEE.

Lian, Y., Shyy, W., Viieru, D., and Zhang, B. (2003). Membrane wing aerodynamics for micro air vehicles. Progress in Aerospace Sciences, 39:425-465.

Lumley, J. L. (1967). The structure of inhomogeneous turbulent fows. Atmospheric turbulence and radio wave propagation, pages 166-178.

Mateescu, D., Scholz, O., and Wang, C. (2012). Aerodynamics of Airfoils at Low Reynolds Numbers in the Proximity of the Ground. In 12th Pan-American Congress of Applied Mechanics, pages $2-7$.

Moon, Y. J., Oh, H.-J., and Seo, J.-H. (2005). Aerodynamic investigation of three-dimensional wings in ground effect for aero-levitation electric vehicle. Aerospace Science and Technology, $9(6): 485-494$.

Mosaad, M. A., Gaafary, M. M., and Amin, I. A. (2011). Effect of Airfoil Camber on WIG Aerodynamic Efficiency. Port Said Engineering Research Journal, 1(1):1-9.

Pelletier, A. and Mueller, T. J. (2000). Low Reynolds Number Aerodynamics of Low-AspectRatio, Thin/Flat/Cambered-Plate Wings. Journal of Aircraft, 37(5):825-832.

Pinnau, R. (2008). Model Reduction via Proper Orthogonal Decomposition. In Model Order Reduction: Theory, Research Aspects and Applications, pages 95-109. Springer Berlin Heidelberg.

Pistolesi, E. (1937). Ground effect - theory and practice. National Advisory Committee for Aeronautics, 828. 
Prasad, R. and Damodaran, M. (2013). Computational Modeling of Steady and Unsteady Low Speed Wing in Ground Effect Aerodynamics. In 51st AIAA Sciences Meeting including the New Horizons Forum and Aerospace Exposition, Dallas, Texas.

Qu, Q., Jia, X., Wang, W., Liu, P., and Agarwal, R. K. (2014). Numerical Simulation of the Flowfield of an Airfoil in Dynamic Ground Effect. Journal of Aircraft, 51(5):1659-1662.

Qu, Q., Wang, W., Liu, P., and Agarwal, R. K. (2015). Airfoil Aerodynamics in Ground Effect for Wide Range of Angles of Attack. AIAA Journal, pages 1-14.

Rad, M. and Kazemi, F. J. (2001). Effect of Camber and Thickness on the Aerodynamic Properties of an Airfoil in Ground Proximity. International Journal of Engineering, 14(3):273-280.

Raymond, A. E. (1921). Ground influence on airfoils . National Advisory Committee for Aeronautics, 67.

Rhodes, S. C. and Sayers, A. T. (2009). Experimental Investigation : Stability Criteria of an Uncambered Airfoil in Ground Effect. $R \&$ D Journal of the South African Institution of Mechanical Engineering, 25:10-15.

Rojratsirikul, P., Genc, M. S., Wang, Z., and Gursul, I. (2011). Flow-induced vibrations of low aspect ratio rectangular membrane wings. Journal of Fluids and Structures, 27:1296-1309.

Rojratsirikul, P., Wang, Z., and Gursul, I. (2009). Unsteady fluid-structure interactions of membrane airfoils at low Reynolds numbers. Experiments in Fluids, 46:859-872.

Rojratsirikul, P., Wang, Z., and Gursul, I. (2010). Effect of pre-strain and excess length on unsteady fluid-structure interactions of membrane airfoils. Journal of Fluids and Structures, $26: 359-376$.

Rozhdestvensky, K. V. (2006). Wing-in-ground effect vehicles. Progress in Aerospace Sciences, 42(3):211-283.

Schmit, R. F., Glauser, M. N., and Gorton, S. A. (2003). Low Dimensional Tools for FlowStructure Interaction Problems: Application to Micro Air Vehicles. In 41st AIAA Aerospace Science Exhibit Meeting and Exhibit, pages 1-9, Reno, NV.

Schreier, H. W., Braasch, J. R., and Sutton, M. A. (2000). Systematic Errors in Digital Image Correlation caused by Intensity Interpolation. Optical Engineering, 39(November 2000):29152921.

Shi, S., New, T., and Liu, Y. (2013). Flapping dynamics of a low aspect-ratio energy-harvesting membrane immersed in a square cylinder wake. Experimental Thermal and Fluid Science, 46:151-161.

Shyy, W., Ifju, P., and Viieru, D. (2005). Membrane Wing-Based Micro Air Vehicles. Applied Mechanics Reviews, 58(7):283-301.

Shyy, W., Klevebring, F., Nilsson, M., Sloan, J., Carroll, B., and Fuentes, C. (1999). Rigid and Flexible Low Reynolds Number Airfoils. Journal of Aircraft, 36(3):523-529. 
Sirovich, L. (1987). Turbulence and the dynamics of coherent structures, I-III. Quarterly of Apploed Mathematics, 45(3):561-590.

Smith, R. and Shyy, W. (1996). Computation of aerodynamic coefficients for a flexible membrane airfoil in turbulent flow: A comparison with classical theory. Physics of Fluids, 8(12):3346-3353.

Stanford, B., Ifju, P., and Albertani, R. (2014). Fixed Membrane Wings for Micro Air Vehicles : Experimental Characterization, Numerical Modeling, and Tailoring. Bret Kennedy Stanford, pages $1-53$.

Stanford, B., Lacore, D., Albertani, R., Parker, G., Walker, R., and Curtis, D. (2010). Proper Orthogonal Decomposition of Flexible Clap and Fling Motions via High-Speed Deformation Measurements. 48th AIAA Aerospace Sciences Meeting, pages 1-18.

Swartz, S., Iriarte-Diaz, J., and Riskin, D. K. (2007). Wing Structure and the Aerodynamic Basis of Flight in Bats. In 45th AIAA Aerospace Sciences Meeting and Exhibit, Reno, NV.

Timpe, A., Zhang, Z., Hubner, J., and Ukeiley, L. (2013). Passive flow control by membrane wings for aerodynamic benefit. Experiments in Fluids, 54(1471):1-23.

Tregidgo, L., Wang, Z., and Gursul, I. (2012). Frequency lock-in phenomenon for self-sustained roll oscillations of rectangular wings undergoing a forced periodic pitching motion. Physics of Fluids, 24(117101):1-18.

Tregidgo, L., Wang, Z., Gursul, I., Kingdom, U., and Student, P. (2011). Fluid-Structure Interactions for a Low Aspect-Ratio Membrane Wing at Low Reynolds Numbers. In 41 st AIAA Fluid Dynamics Conference and Exhibit, number June, Honolulu,Hawai.

Van Beek, C. and Oskam, B. (1998). Progress Report on Aerodynamic Analysis of a Surface Piercing Hydrofoil-Controlled Effect Seabus Configuration. In RTO AVT Symposium, volume 23, pages 23-1 to 23-18, Amsterdam. National Aerospace Laboratory.

Visbal, M., Gordnier, R. E., and Galbraith, M. (2009). High-fidelity simulations of moving and flexible airfoils at low Reynolds numbers. Experiments in Fluids, 46:903-922.

Vogt, J. and Barber, T. (2012). Ground effect phenomena about lift and downforce generating cambered aerofoils. International Journal of Numerical Methods for Heat $\&$ Fluid Flow, $22(2): 153-174$.

Wieselsberger, C. (1921). Wing Resistance Near the Ground. Technical report, Zeitschrift für Flugtechnik und Motorluftschiffahrt.

Yun, L., Bliault, A., and Doo, J. (2010). WIG Craft and Ekranoplan: Ground Effect Craft Technology. In Springer, ISBN: 9781441900425.

Zhang, X. and Zerihan, J. (2003). Off-Surface Aerodynamic Measurements of a Wing in Ground Effect. Journal of Aircraft, 40(4):716-725. 